\title{
Analysis of Peripheral Vision and Vibrotactile Feedback During Proximal Search Tasks with Dynamic Virtual Entities in Augmented Reality
}

\author{
Kendra Richards* \\ University of Central Florida \\ Orlando, Florida \\ kendrarichards230@gmail.com \\ Ryan Schubert \\ University of Central Florida \\ Orlando, Florida \\ rschuber@ist.ucf.edu \\ Nahal Norouzi \\ University of Central Florida \\ Orlando, Florida \\ nahal.norouzi@knights.ucf.edu
}

\author{
Nikhil Mahalanobis* \\ University of Central Florida \\ Orlando, Florida \\ nmahalanobis@knights.ucf.edu \\ Myungho Lee \\ University of Central Florida \\ Orlando, Florida \\ myungho@knights.ucf.edu \\ Jason Hochreiter \\ University of Central Florida \\ Orlando, Florida \\ jhochrei@cs.ucf.edu \\ Gregory F. Welch \\ University of Central Florida \\ Orlando, Florida \\ welch@ucf.edu
}

\author{
Kangsoo Kim \\ University of Central Florida \\ Orlando, Florida \\ kangsoo.kim@ucf.edu \\ Salam Daher \\ University of Central Florida \\ Orlando, Florida \\ salam.daher@ucf.edu \\ Gerd Bruder \\ University of Central Florida \\ Orlando, Florida \\ bruder@ucf.edu
}

\begin{abstract}
A primary goal of augmented reality (AR) is to seamlessly embed virtual content into a real environment. There are many factors that can affect the perceived physicality and co-presence of virtual entities, including the hardware capabilities, the fidelity of the virtual behaviors, and sensory feedback associated with the interactions. In this paper, we present a study investigating participants' perceptions and behaviors during a time-limited search task in close proximity with virtual entities in AR. In particular, we analyze the effects of (i) visual conflicts in the periphery of an optical see-through head-mounted display, a Microsoft HoloLens, (ii) overall lighting in the physical environment, and (iii) multimodal feedback based on vibrotactile transducers mounted on a physical platform. Our results show significant benefits of vibrotactile feedback and reduced peripheral lighting for spatial and social presence, and engagement. We discuss implications of these effects for AR applications.
\end{abstract}

*Both authors contributed equally to this research.

Permission to make digital or hard copies of all or part of this work for personal or classroom use is granted without fee provided that copies are not made or distributed for profit or commercial advantage and that copies bear this notice and the full citation on the first page. Copyrights for components of this work owned by others than ACM must be honored. Abstracting with credit is permitted. To copy otherwise, or republish, to post on servers or to redistribute to lists, requires prior specific permission and/or a fee. Request permissions from permissions@acm.org.

SUI '19, October 19-20, 2019, New Orleans, LA, USA

(c) 2019 Association for Computing Machinery.

ACM ISBN 978-1-4503-6975-6/19/10 . \$ \$15.00

https://doi.org/10.1145/3357251.3357585

\section{CCS CONCEPTS}

- Human-centered computing $\rightarrow$ User studies; Mixed / augmented reality.

\section{KEYWORDS}

Augmented Reality; Field of View; Multimodal Feedback; Search Task

ACM Reference Format:

Kendra Richards, Nikhil Mahalanobis, Kangsoo Kim, Ryan Schubert, Myungho Lee, Salam Daher, Nahal Norouzi, Jason Hochreiter, Gerd Bruder, and Gregory F. Welch. 2019. Analysis of Peripheral Vision and Vibrotactile Feedback During Proximal Search Tasks with Dynamic Virtual Entities in Augmented Reality. In Symposium on Spatial User Interaction (SUI '19), October 19-20, 2019, New Orleans, LA, USA. ACM, New York, NY, USA, 9 pages. https://doi.org/10.1145/3357251.3357585

\section{INTRODUCTION}

Augmented reality (AR) technologies have shown great potential for a wide range of application fields from in-situ interior design to entertainment and games, and recent trends project even more widespread proliferation in the near future [37]. Compared to virtual reality (VR) environments, such AR setups are characterized by users being able to see both the real world and virtual content superimposed or embedded among the physical environment. For instance, optical see-through head-mounted displays (OST-HMDs) create the illusion of a shared physical-virtual space by tracking the user's head pose relative to the physical environment, e.g., using SLAM [11], and presenting registered virtual geometry as part of the user's visual field. However, current-state OST-HMDs are 
limited by different factors ranging from the weight and comfort of the head-mounted sensor and display hardware to the fidelity of the visual and extraretinal sensory feedback [18, 21, 23].

In this paper, we focus on two primary limitations that are prevalent in current AR HMD setups:

(1) The augmented visual region is limited to a small central area, i.e., a narrow field of view, which can lead to a physicalvirtual information conflict between the central and peripheral view, and cause unnaturally cropped or visually absent virtual content.

(2) The sensory stimulation is limited to visual and audio augmentation, which can lead to conflicts due to cross-modal causalities and correlations experienced in the real world.

In this paper, we discuss and evaluate these limitations with a Microsoft HoloLens in the scope of a gamified search task in close proximity of dynamic virtual objects. First, we compare two display conditions in which we either present an unrestricted but unaugmented periphery or an occluded periphery using a physical lightshield (inspired by Lee et al.'s study [23]). Second, we evaluate effects of the lighting conditions in the physical environment with respect to light concentrated in front to illuminate the central view or reaching into the periphery. Third, we analyze two feedback conditions using a physical platform in which we either present vibrotactile feedback synchronized with the movement of virtual entities or no vibrotactile feedback (inspired by Lee et al.'s study [24]).

In particular, we address the following research questions:

RQ1 Can a lightshield that occludes the unaugmented periphery of the OST-HMD increase co-presence and compensate for the absence of virtual information in the periphery?

RQ2 Can vibrotactile feedback on the floor increase co-presence and compensate for a limited visual field?

RQ3 Does the absence of peripheral vision in the OST-HMD induce similar co-presence as the absence of light in the periphery of the user's eyes in the physical environment?

This paper is structured as follows. Section 2 presents an overview of related work. Section 3 describes the human-subject study. The results are presented in Section 4 and discussed in Section 5. Section 6 concludes the paper and discusses future research.

\section{RELATED WORK}

In this section, we resume related work with respect to the sense of co-presence of virtual entities in AR, the field of view of HMDs, and haptic feedback.

\subsection{Co-Presence}

The term presence in the VR literature usually refers to one's sense of "being there" in a virtual environment. In AR, the concept of copresence might be described as how one perceives another entity's presence in a sense of "being together" [38]. In a social context, Harms and Biocca [6] characterized co-presence as one of several sub-dimensions that make up social presence $[8,9]$.

Slater introduced an important concept related to presence and co-presence, called plausibility illusion (Psi) [34]. Psi refers to the "illusion that the scenario being depicted is actually occurring," which "requires a credible scenario and plausible interactions between the participant and objects and virtual characters in the environment."

Considering the definitions addressed above, we expect that the fidelity and plausibility of the visual and haptic feedback in AR setups, in particular when using OST-HMDs, could be important factors shaping the user's sense of co-presence.

\subsection{Field of View}

The total human visual field extends over about 200 degrees horizontally and 135 degrees vertically [29]. The central region of the visual field is most important for daily tasks due to its visual acuity, stereopsis, and related depth cues. However, the human visual periphery is important for recognizing structure, shapes, maintaining body posture, movement speed, and heading among others [29]. Alfano and Michel showed that peripheral vision is important for situational awareness [1]. Reduced peripheral vision can lead to behavioral changes, e.g., increasing the frequency and amplitude of head rotations to compensate for the reduced amount of information that can be taken in at any time $[2,14]$. Toet et al. showed that restricted peripheral vision can increase the difficulty of navigation and wayfinding tasks, resulting in significantly more time needed to traverse a complex environment [35, 36]. The limited field of view was also identified as a potential factor affecting distance underestimation in immersive HMDs [28, 32], with benefits shown for distance judgments and natural gait for larger fields of view $[16,17]$.

Related to search tasks in VR, Ragan et al. further found that the field of view had an effect on target detection performance, with overall negative effects when the HMD's field of view was reduced [30]. Ren et al. showed similar effects of the field of view of AR HMDs on visual search task performance [31]. Similar to our approach, the work done by Lee et al. suggested that restriction of peripheral vision while wearing a HoloLens increases the amount of head movements and changes proxemic behavior with real and virtual humans, but they did not find significant effects in support of increased co-presence with virtual entities in AR [23].

\subsection{Haptic Feedback}

Haptic feedback is an important enabling factor for the sense of co-presence during interactions between real or virtual people or objects in AR and VR. Due to technological challenges, research on haptic feedback in AR/VR environments was very limited and largely absent in the past [10]. Recently, however, there have been increasing research in this area for various vibrotactile and haptic devices.

Basdogan et al. showed that haptic sensations when interacting with other people, such as when moving a virtual object by two persons, increased the sense of co-presence as well as task performance [4]. Sallnäs showed that haptic feedback from a virtual object can increase presence, social presence, and the perceived performance when persons in two remote places passed a virtual object in a shared virtual environment [33]. In a mixed reality environment, Kotranza et al. showed that a simulated medical patient elicited more natural behavior in trainees in a healthcare training context when the patient could be touched, responded to touch, and touched back [20]. Bickmore et al. found positive effects on arousal 
and valence for similar haptic feedback in simulated patients [5] Huisman et al. used vibrotactile feedback during touch interaction in an AR setup, and showed that it changed affective adjectives [15].

Recently, researchers found positive effects of indirect haptic feedback, i.e., when a user is not directly touching a virtual object or entity but a shared intermediate object induces stimuli that create an illusion of co-presence. In particular, Lee et al. found that subtle movements of a "Wobbly Table" caused by mechanical actuators could significantly increase co-presence when communicating with a virtual human sitting at the other end of the table [25], and when the virtual human could exert forces on physical objects on the table [26]. They further showed that a haptic transducer mounted on the floor could induce vibrotactile feedback synchronized with a virtual human's footsteps, which they found can increase social presence in VR [24]. Similar to our approach, they further applied this concept to an AR environment by transmitting vibrotactile feedback through a physical platform, which they used to increase co-presence with virtual humans in AR [23].

\section{EXPERIMENT}

In this section we describe the experiment that we conducted to investigate the three research questions stated in Section 1. Participants experienced an AR search task, in which they had to search through boxes presented on each shelf of a physical bookcase until they found their target object (in our case a missing kitten) While doing so, participants uncovered other dynamic entities in the boxes, such as spiders or rats jumping out in their direction or bats flying towards them. We varied the visual field in the display, the lighting conditions in the physical environment, and the presence of vibrotactile feedback delivered through a physical platform that shook underneath the participants' feet when one of the virtual entities landed on it and scurried away.

\subsection{Participants}

We recruited 22 participants for our experiment; 13 male and 9 female (ages 18 to $41, M=26.1, S D=5.90$ ). The participants were members of the local university community. All of the participants had normal or corrected-to-normal vision; three participants wore glasses during the experiment, and six wore contact lenses. Two of the participants reported vision problems (one for Astigmatism and the other for uneven eye dominance), but the rest did not have known visual or vestibular disorders, such as color or night blindness, dyschromatopsia, or a displacement of balance. We also measured the height of each participant $(M=1.71 \mathrm{~m}, \mathrm{SD}=0.10 \mathrm{~m})$. We asked our participants to report any known phobias; six participants reported arachnophobia (fear of spiders), four participants reported musophobia (fear of mice or rats), and two reported nyctophobia (fear of the dark).

\subsection{Material}

3.2.1 Physical Bookcase with Virtual Entities. We placed a bookcase with three layers of shelves in front of the participants (see Figure 1). Each shelf was filled with four cardboard boxes. The dimensions of the bookcase were $0.764 \mathrm{~m}$ (width) $\times 1.467 \mathrm{~m}$ (height) $\times 0.391 \mathrm{~m}$ (depth). We further placed a 10" CCTV LED screen on top of the bookcase and we connected it to a workstation to present a timer that counted down from 60 seconds and indicated the remaining time to complete the task.

Each box had a size of $0.164 \mathrm{~m}$ (width) $\times 0.252 \mathrm{~m}$ (height) $\times$ $0.368 \mathrm{~m}$ (depth). We placed common labels and stickers on the boxes, such as "Keep Dry" or "Caution Heavy" which were chosen to be nondescript, similar, randomly distributed, and not related to the virtual stimuli.

We used different virtual characters that were modeled, rigged, and textured in Autodesk Maya as well as animated and rendered in the Unity game engine. The virtual stimuli consisted of the following types of dynamic entities that could be encountered by participants in the experiment:

- Barbara: 3D virtual human with a green shirt and blue jeans, who took on the image of an adult female, appeared next to the bookcase at the beginning of the task while opening up a virtual sliding door. She talked to participants by telling her name, Barbara, and explained about her missing cat. She requested help to look for her cat, which could be inside one of the boxes in the bookcase, and then disappeared. To make her request for assistance was more plausible, she was seated in a wheelchair.

- Kitty: a virtual juvenile cat with orange and yellow fur was sitting in one of the boxes in the bookcase. The kitty meowed when its box was opened.

- Creatures: we prepared four different types of virtual creatures that hid inside the boxes in the bookcase: bats, rats, spiders, and scorpions. These creatures were randomly placed in the boxes.

- Bat: a black and brown bat flew out of a box while making fluttering sounds with its wings when participants opened the box.

- Rat: a gray rat jumped out of a box with a squeaking sound towards the participant, and landed on the platform where the participant was standing on.

- Spider: a big black spider jumped out of a box towards the participant without any sound, and landed on the platform where the participant was standing on.

- Scorpion: a big gray scorpion jumped out of a box towards the participant without any sound, and landed on the platform where the participant was standing on.

The occurrences of the virtual entities were randomized. However, only up to one kitty was presented per trial as participants were instructed to look for the kitty as the search target. Some illustrations of the virtual entities in AR from the participant's viewpoint are shown in Figure 2.

3.2.2 Vibrotactile Platform. As shown in Figure 1, participants were instructed to stand on a solid wooden platform with dimensions, $1.26 \mathrm{~m}$ (length) $\times 0.913 \mathrm{~m}$ (width). Because the platform was about $4 \mathrm{~cm}$ raised from the floor, we added safety lines around the platform (see Figure 1). To induce visually synchronized vibrations for the impact and movements of the virtual entities on the platform, we mounted a ButtKicker $\mathrm{LFE}^{1}$, a low-frequency vibrotactile transducer, on a corner of the platform as shown in the figure. The transducer was able to shake the entire platform based on audio

\footnotetext{
${ }^{1}$ http://www.thebuttkicker.com
} 


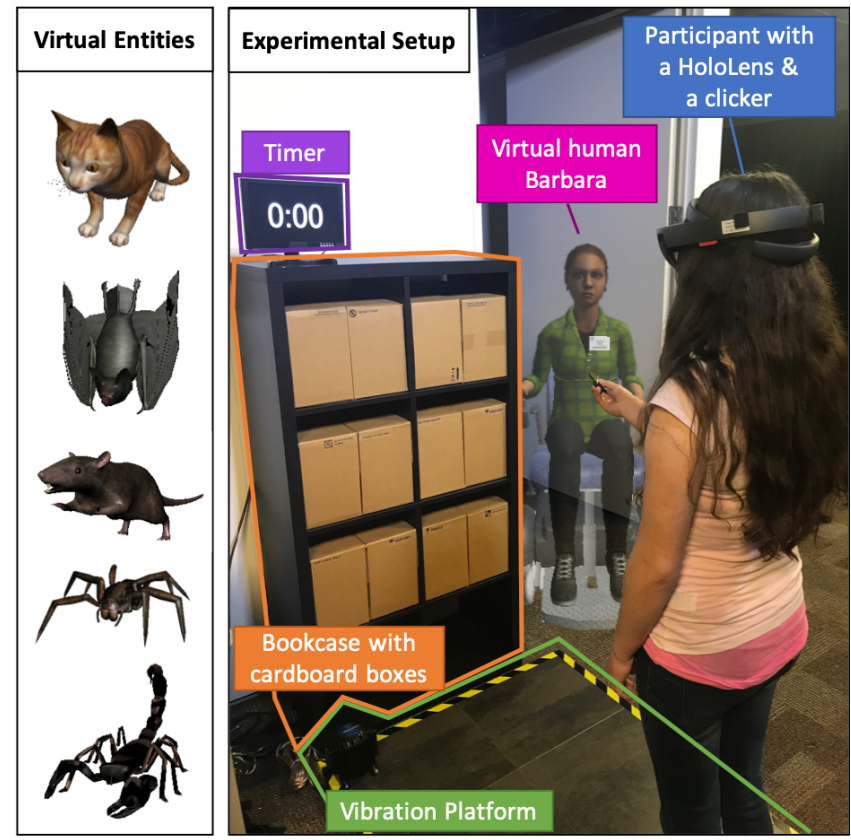

Figure 1: Photo of the experimental setup with annotations. The left side shows the different types of virtual entities, which are placed in the boxes on the shelves, and the right side shows the AR setup with the physical components and a virtual human.
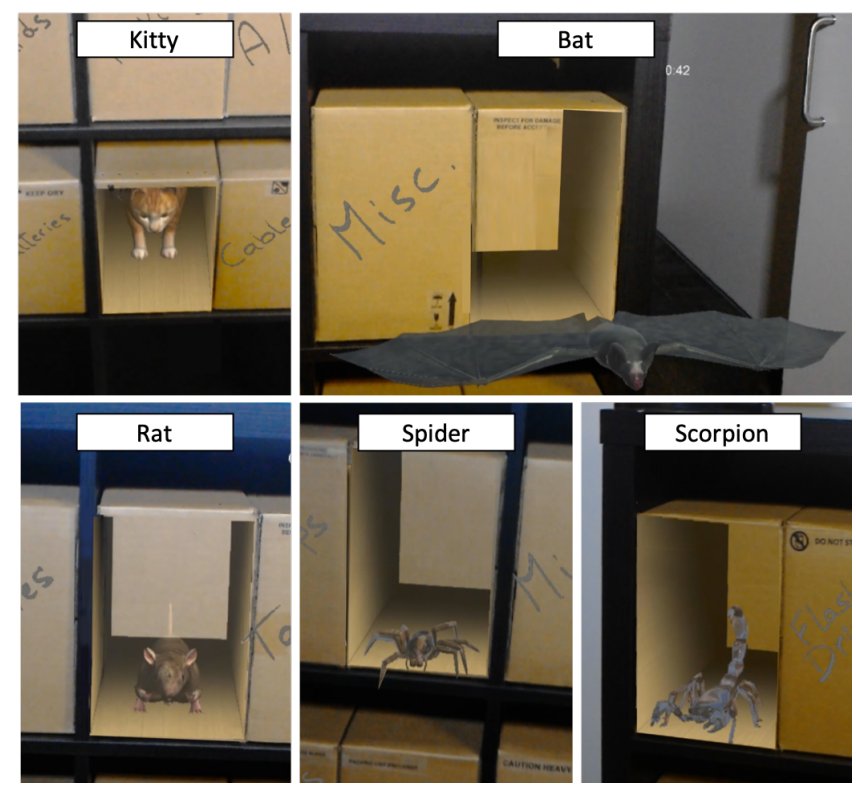

Figure 2: Illustration of the physical boxes in the experiment with a AR virtual kitty and creatures jumping or flying out toward the participant. Images were captured through the HoloLens device portal. input received from a connected MSI VR ONE 7RE-065US Virtual Reality Backpack PC , and amplified with a ButtKicker Power Amplifier BKA1000-4A.

To generate the vibrations, we used a client-server model. The client was running on a Microsoft HoloLens and rendered the virtual entities. When a virtual object jumped out of a box and onto the platform, we determined the collision and sent a message to the server, which the vibrotactile transducer was connected to. The server then played a pre-recorded low-frequency sound, i.e., an impact sound on a wooden floor with a low-pass filter applied, for the impacts, which was transmitted to the transducer through the amplifier. We used a similar sound for the "scurrying away" feedback when a virtual entity moved over the platform after the impact to get away. Because we had a fixed setup with known vertical distances to the platform, we could slightly adjust the collision object to counteract any pre-measured latency between the virtual entity and the resulting vibrations. The client-server communication was realized through the Unity HLAPI, and both were connected to an ASUS RT-AC5300 high-speed router. This process ensured that the visual cues on the HoloLens and the vibrotactile cues on the platform were synchronized.

The two considered vibrotactile conditions were as follows:

- Vibration On: Participants felt the impact and movements of virtual content through the platform.

- Vibration Off: No vibrotactile feedback was presented.

3.2.3 Head-Mounted Display. We made the decision to use a Microsoft HoloLens for this experiment. As a widely-used OST-HMD, the HoloLens provides a narrow (circa 30 degrees horizontally by 17 degrees vertically) augmented field of view (FOV) in the center of the total human visual field (see Section 2.2). A person wearing the HoloLens usually perceives a small augmented central region and a large unaugmented visual field in the periphery of the display. This means that if a virtual object is larger than the augmented central region, it progressively disappears as it passes into the unaugmented periphery, which is particularly challenging for small dynamic virtual content that is located close to the observer. Lee et al. discussed that such disappearing visual information does not naturally occur in normal human vision, and that related visual conflicts may negatively affect the overall AR experience [23]. In order to avoid such a conflict, it is possible to cover the periphery of the HoloLens with a thin physical lightshield that attaches to the inner side of the visor and blocks the unaugmented periphery. While it is not an optimal solution in that it reduces the overall FOV, the benefits are solely that the visual conflicts between the virtual content and the physical environment are resolved.

The two considered viewing setups in this experiment were therefore as follows (see Figure 3):

- Unrestricted FOV: Both the augmented central field and the unaugmented periphery were visible.

- Restricted FOV: The unaugmented periphery was occluded with a physical lightshield such that only the augmented central region was visible.

3.2.4 Physical Lighting. To evaluate the differences between occluding the periphery of the OST-HMD and the absence of light in the physical environment, we controlled the overall amount of light 


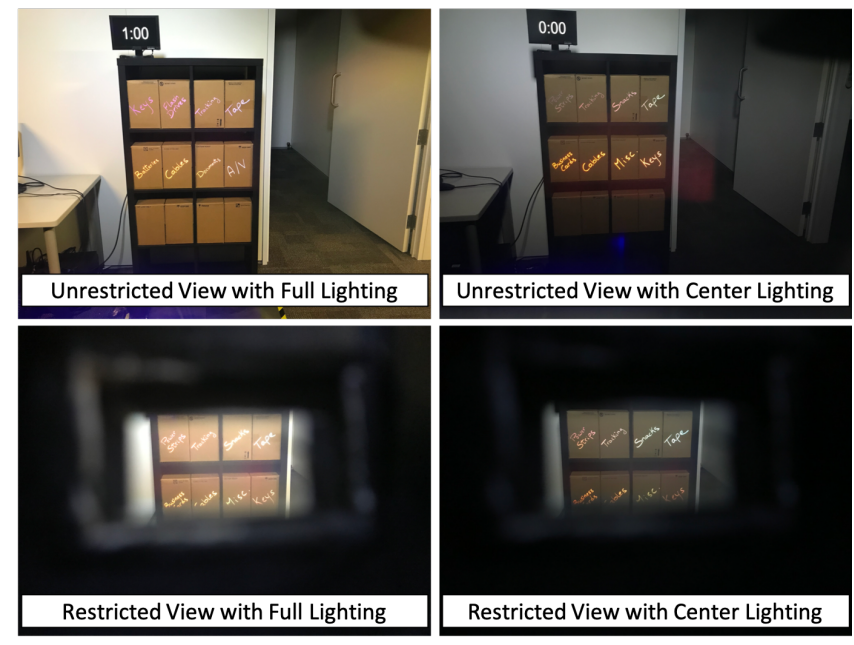

Figure 3: Examples of participant views regarding the peripheral view and physical lighting. Rows: unrestricted view (first) and restricted view (second) conditions. Columns: full lighting (first) and center lighting (second) conditions. Images were captured directly through the HoloLens display.

in the experimental setup. We created a well-lit environment that illuminated not only the bookcase in front of the participants but also the floor and periphery. In contrast, we reduced the lighting to only illuminate the bookcase in front of the participants, while leaving the floor and the periphery in shadow. We expected to see increased benefits of the vibrotactile feedback on co-presence in the cases when the periphery was hidden in shadows and/or occluded through the lightshield.

The two considered lighting conditions in this experiment were as follows (see Figure 3):

- Full Lighting: Both the bookcase in front and the periphery were well-lit due to diffuse ceiling lighting.

- Central Lighting: Only the bookcase in front was illuminated due to spot lighting from the ceiling, while the periphery was hidden in shadows.

\subsection{Methods}

We chose to use a full-factorial within-subjects design for this experiment due to the number of conditions and expected interpersonal differences in behavior [13]. The independent variables were as follows:

- Peripheral View (Restricted, Unrestricted),

- Vibrotactile Feedback (On, Off), and

- Physical Lighting (Full, Central).

Table 1 summarizes the conditions, and Figure 3 shows examples of participant view with respect to the peripheral view and physical lighting factors. We decided to use a randomized block design with the lighting conditions as blocking factors due to the time overhead for changing the lighting conditions in the experiment, i.e., we tested these conditions as a block, but we randomized the order of the blocks as well as the conditions that were tested within the block.
Table 1: Study conditions: Each column refers to factors we controlled. The rows indicate the tested combinations.

\begin{tabular}{|c|c|c|}
\hline Lighting & View & Vibration \\
\hline \multirow{3}{*}{ Full } & \multirow{2}{*}{ Unrestricted } & On \\
\cline { 3 - 3 } & & Off \\
\cline { 3 - 3 } & \multirow{2}{*}{ Restricted } & On \\
\cline { 3 - 3 } Central & \multirow{2}{*}{ Unrestricted } & Off \\
\cline { 3 - 3 } & & On \\
\cline { 3 - 3 } & \multirow{2}{*}{ Restricted } & Off \\
\cline { 3 - 3 } & & On \\
\hline
\end{tabular}

Each participant completed eight conditions in the randomized block design.

3.3.1 Procedure. Prior to the experiment trials, participants first were asked to give their informed consent. Afterwards, they received task instructions and the experimenters made sure that they understood the task.

At the beginning of each trial, participants were instructed to stand on the designated platform, right in front of the bookcase with the boxes on the shelves (Figure 1). The participants were then shown how to properly put on the Microsoft HoloLens. After they adjusted the HoloLens on their head, the first trial started by seeing a virtual human entering the physical space on the right side of the bookcase. They then heard the virtual human, a rigged and animated female character in a wheelchair called "Barbara," give them their verbal task instructions. The participants were thus instructed to search for her lost kitty by looking through the boxes on the shelves. After these instructions, the virtual human moved away and the timer stated on the bookcase. At this point, the participants started opening one box at a time by using the HoloLens' clicker and gaze selection, i.e., we asked them to orient the HoloLens such that the box was in their view and press the clicker. This was then followed by a virtual animation showing the box opening in front of them, which then revealed one of the virtual entities inside that are described in Section 3.2.1. As mentioned before, that entity would then potentially come jumping out of the box in the participant's direction, hitting the floor near them, which then caused vibrotactile feedback in some of the conditions. The trial ended either after the participant found the kitty, which was located in one of the twelve boxes, or the 60-second timer ran out. We randomized the locations of the virtual entities between trials, but we made sure that the kitty could only be found in the second half of the total time to make sure that they experienced at least a few of the visual-vibrotactile effects before the trial ended. During the trial, we recorded the participants' head position and orientation, and one of the experimenters coded their avoidance behaviors. After each trial, participants were guided to complete questionnaires that measures their subjective perception of the experience. Once completing all eight trials, participants filled out post-experiment questionnaires consisting of demographics data and open questions, and participants finished the study by receiving monetary compensation.

3.3.2 Behavioral Measures. While performing the search task, participants' head position and orientation were continuously tracked by a professional OptiTrack Trio tracking system, and one of the 
Table 2: The subjective Spatial Presence questionnaire.

\begin{tabular}{c|l}
\hline ID & Question \\
\hline SP1 & $\begin{array}{l}\text { How much did it seem as if the animals you saw/heard had come to the } \\
\text { place you were? }\end{array}$ \\
\hline SP2 & $\begin{array}{l}\text { How much did it seem as if you could reach out and touch the animals } \\
\text { you saw/heard? }\end{array}$ \\
\hline SP3 & $\begin{array}{l}\text { How often when the animal seemed to be headed toward you did you } \\
\text { want to move to get out of its way? }\end{array}$ \\
\hline SP4 & $\begin{array}{l}\text { To what extent did you experience a sense of being there in the envi- } \\
\text { ronment with the animals you saw/heard? }\end{array}$ \\
\hline SP5 & $\begin{array}{l}\text { To what extent did it seem that sounds came from specific different } \\
\text { locations? }\end{array}$ \\
\hline SP6 & How often did you think the animals you saw/heard can touch you? \\
\hline SP7 & $\begin{array}{l}\text { Did the experience seem more like looking at the animals on a movie } \\
\text { screen or more like looking at the animals through a window? }\end{array}$ \\
\hline
\end{tabular}

experimenters were coding their body behavior. We logged the movements relative to the orientation of the platform in front of the bookcase. We considered the following dependent variables:

- Head Rotations: We computed the amount of head rotation of the participant by calculating the standard deviation of their head orientation along the yaw and pitch axes, which allows us to understand if they were more inclined to rotate their head left or right, or look down at the floor, in any of the conditions.

- Avoidance Behavior: We observed participants' avoidance behavior with their head and body when the virtual entities came towards them, and we classified them into three groups: (a) pulling back in sagittal direction from the virtual entities, (b) moving sideways in lateral direction out of their way, and/or (c) raising their hands to protect themselves from the virtual entities.

3.3.3 Subjective Measures. We measured participants' perception of the virtual stimuli via questionnaires and post-trial surveys. Leveraging items from standard questionnaires, we created a more focused post-trial survey based on 7-point multi-item scales in four dimensions:

- Spatial Presence: This dimension is about the sense of presence in which the participants feel that they or other objects (in our case virtual entities), or environments have been transported. In other words, the concept includes the sense of "you are there." We used seven related questions adapted from those used by Lombard et al. [27]. See Table 2.

- Social Presence: We used five related questions from Bailenson et al. [3] for social presence. The questions tend to cover the perceived authenticity of the virtual entities as well as the sense of "being together" with the entities. See Table 3.

- Engagement: We also measured how engaging the experience was using six related questions from Lombard et al. [27] See Table 4.

- Gaming Experience: Considering the search task in the experiment as a gaming experience, we also used nine related questions that we adapted from Calvillo Gámez et al. [12] See Table 5.

We further asked our participants to indicate if they have any phobias; specifically, phobias of the animals that are represented by the virtual entities in this experiment, and a phobia of darkness.
Table 3: The subjective Social Presence questionnaire. Responses from questions marked with a "*” were inverted for the analysis.

\begin{tabular}{c|l}
\hline ID & Question \\
\hline SO1 & I perceive that I am in the presence of the animals in the room with me. \\
\hline SO2 & I feel that the animals are watching me and are aware of my presence. \\
\hline SO3 $^{*}$ & $\begin{array}{l}\text { The thought that the animals are not real animals crosses my mind } \\
\text { often. }\end{array}$ \\
\hline SO2 & The animals appear to be sentient, conscious, and alive to me. \\
\hline SO3 $^{*}$ & $\begin{array}{l}\text { I perceive the animals as being only computerized images, not as real } \\
\text { animals. }\end{array}$ \\
\hline
\end{tabular}

Table 4: The subjective Engagement questionnaire.

\begin{tabular}{c|l}
\hline ID & Question \\
\hline EN1 & To what extent did you feel mentally immersed in the experience? \\
\hline EN2 & How fun was the experience? \\
\hline EN3 & How completely were your senses engaged? \\
\hline EN4 & To what extent did you experience a sensation of reality? \\
\hline EN5 & How relaxing or exciting was the experience? \\
\hline EN6 & How engaging was the story? \\
\hline
\end{tabular}

Table 5: The subjective Gaming Experience questionnaire. Responses from questions marked with a “*” were inverted for the analysis.

\begin{tabular}{c|l}
\hline ID & Question \\
\hline GE1 & I enjoyed playing the game \\
\hline GE2 & I would play this game again \\
\hline GE3 & There was time when I was doing nothing in the game. \\
\hline GE4 & I liked the way the game looks. \\
\hline GE5* & I got bored playing this time. \\
\hline GE6 & I felt what was happening in the game was my own doing. \\
\hline GE7 & The graphics were appropriate for the type of game. \\
\hline GE8 & The sound effects of the game were appropriate. \\
\hline GE9 & The scenario of the game was interesting. \\
\hline
\end{tabular}

We compare these phobias to the other results of the experiment as we believe that participants who have a phobia of one of the creatures are more likely to have feelings of anxiety, stress, or fear, which can influence their responses.

\subsection{Hypotheses}

Based on the related work, and our study design, we formulated the following hypotheses for the reactions of participants:

H1 Participants will show higher co-presence on the questionnaires and in terms of the behavioral measures for the condition with vibrotactile feedback.

H2 Participants will indicate higher subjective measures of copresence when the lighting is limited to the central region.

H3 Participants will indicate higher subjective ratings and behavioral effects associated with co-presence with the restricted field of view.

\section{RESULTS}

We first present the descriptive and inferential statistical analysis of the subjective questionnaire responses, followed by the behavioral measures. We used parametric statistical tests to analyze the responses in line with the ongoing discussion in the field of psychology indicating that parametric statistics can be a valid and more 


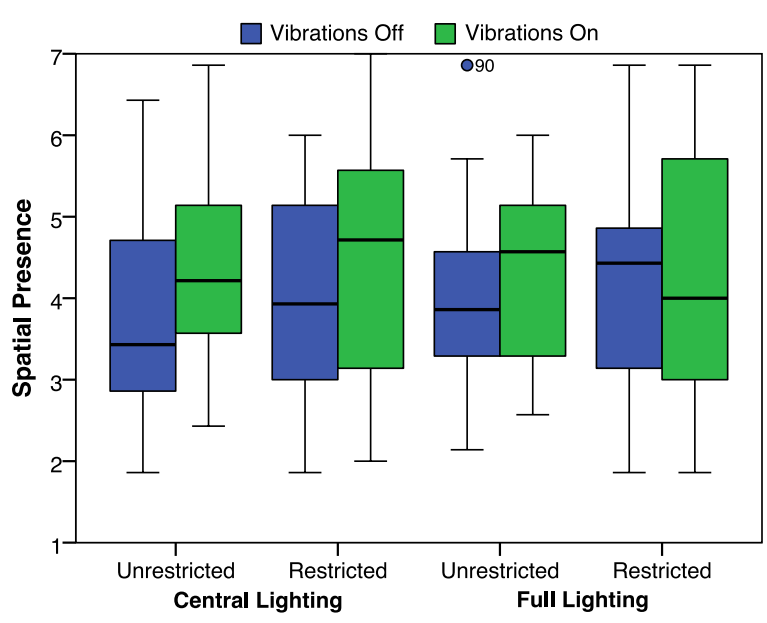

(a)

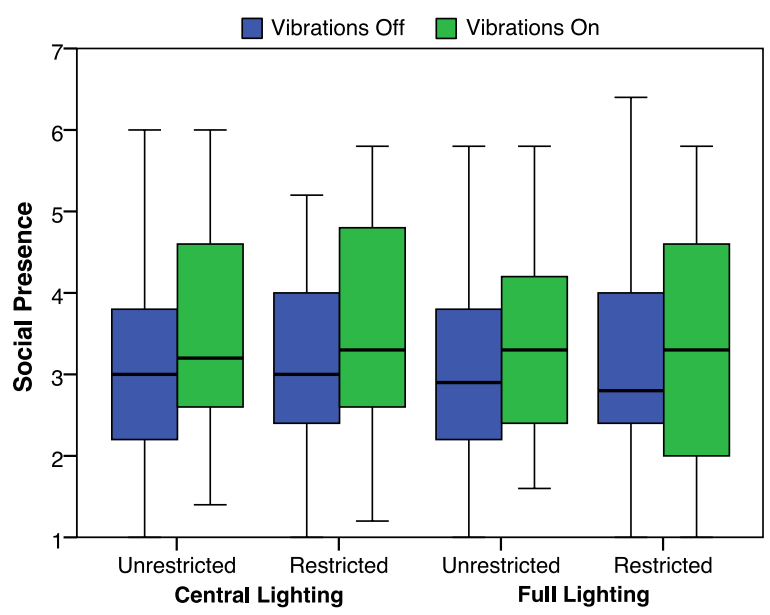

(c)

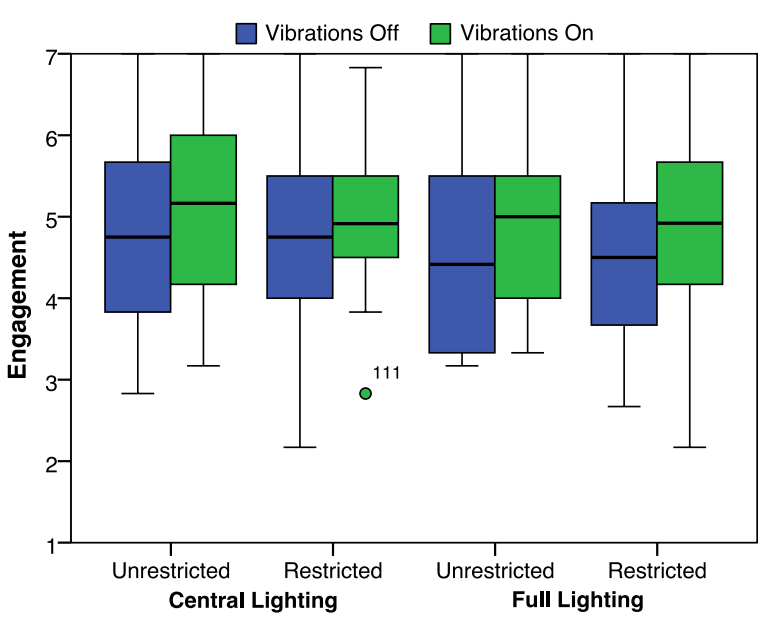

(b)

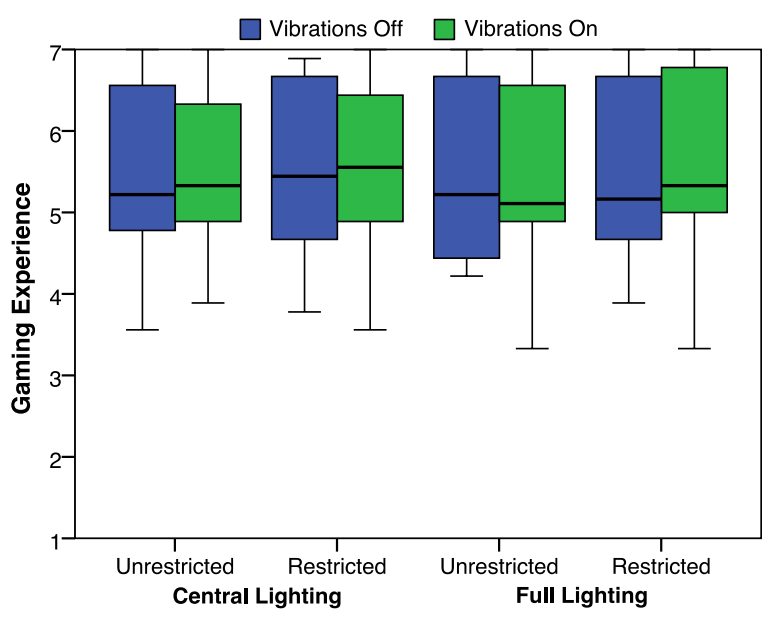

(d)

Figure 4: Results for (a) spatial presence, (b) engagement, (c) social presence, and (d) gaming experience.

informative method for the analysis of combined experimental questionnaire scales with individual ordinal data points measured by questionnaires or coded behaviors [19, 22]. We analyzed the responses with repeated-measures ANOVAs and Tukey multiple comparisons with Bonferroni correction at the $5 \%$ significance level. We confirmed the normality with Shapiro-Wilk tests at the 5\% level and QQ plots. Degrees of freedom were corrected using GreenhouseGeisser estimates of sphericity when Mauchly's test indicated that the assumption of sphericity had been violated. We only report the significant effects.

\subsection{Subjective Measures}

The results for spatial presence, engagement, social presence, and gaming experience are shown in Figure 4. The $x$-axes show the conditions. The $y$-axes show the pooled mean responses. Results for vibrotactile feedback are shown in green and without feedback in blue.
We found a significant main effect of the vibrotactile feedback on spatial presence, $F(1,21)=14.33, \boldsymbol{p}=\mathbf{0 . 0 0 1}, \eta_{p}^{2}=0.406$, as well as on social presence, $F(1,21)=6.73, \boldsymbol{p}=\mathbf{0 . 0 1 7}, \eta_{p}^{2}=0.243$, and also on engagement, $F(1,21)=5.25, \boldsymbol{p}=\mathbf{0 . 0 3 2}, \eta_{p}^{2}=0.200$, indicating that the vibrations positively affected participants' sense of spatial presence, social presence, as well as engagement.

We found a significant main effect of physical lighting on engagement, $F(1,21)=4.43, \boldsymbol{p}=\mathbf{0 . 0 4 8}, \eta_{p}^{2}=0.174$, showing that participants felt more engaged if the lighting in the room was reduced to the central area illuminating the bookcase in front of them and did not reach into the periphery.

We found no significant main effect of peripheral view and no interaction effect for any combination of independent variables and dependent variables.

We also found no effect of the phobias the participants reported on the results, which we analyzed as a between-subjects factor. 


\subsection{Behavioral Measures}

We found no significant effects of the factors on avoidance behavior that the experimenters coded during the trials. Overall, participants in $4.6 \%$ of the trials showed avoidance behavior in that they pulled their head/torso back from the virtual entities (sagittal direction), and $12.5 \%$ showed sideways avoidance behavior with their head/torso (lateral direction). None of the participants raised their hands in an attempt to protect themselves from the virtual entities.

Regarding the head rotations, for yaw angles, we found no significant effect between the restricted $\left(M=-15.18^{\circ}, S D=15.47^{\circ}\right)$ and unrestricted $\left(M=-15.64^{\circ}, S D=14.67^{\circ}\right)$ view conditions; the central lighting $\left(M=-15.51^{\circ}, S D=15.22^{\circ}\right)$ and full lighting $\left(M=-15.97^{\circ}, S D=14.93^{\circ}\right)$ conditions; or the vibration $(M=$ $\left.-15.58^{\circ}, S D=15.12^{\circ}\right)$ and no-vibration $\left(M=-15.24^{\circ}, S D=15.03^{\circ}\right)$ conditions. Also, we found no significant effect for pitch angles between the restricted $\left(M=9.82^{\circ}, S D=11.76^{\circ}\right)$ and unrestricted $\left(M=10.63^{\circ}, S D=11.85^{\circ}\right)$ view conditions; the central lighting $\left(M=9.75^{\circ}, S D=11.74^{\circ}\right)$ and full lighting $\left(M=10.23^{\circ}, S D=11.88^{\circ}\right)$ conditions; or the vibration $\left(M=10.09^{\circ}, S D=12.07^{\circ}\right)$ and novibration $\left(M=9.90^{\circ}, S D=11.54^{\circ}\right)$ conditions.

\section{DISCUSSION}

In this section, we summarize the main findings and discuss implications for AR applications with current-state OST-HMDs.

\subsection{Vibrotactile Feedback Increases Spatial and Social Presence and Engagement}

In line with our Hypothesis H1, we found that the vibrotactile feedback resulted in a significant increase in spatial presence, social presence, and engagement. It is interesting to see that the vibrations had such a noticeable effect on participants' perception of the virtual entities considering that they only provided subtle stimuli in our experiment compared to the more dominant visual feedback. Most of our participants indicated during the debriefing that the vibrations had a positive effect. As a guideline for practitioners in the field of AR, we suggest adding vibrations through the floor using a floor- or platform-mounted transducer as it is a comparatively easy and low-cost addition compared to other types of haptic feedback, and it seems like a viable option for a range of different types of AR applications.

\subsection{Reduced Peripheral Lighting Increases Engagement}

Although we did not see the effect of lighting in co-presence, in line with our Hypothesis H2, we found a significant increase in engagement when participants were in the restricted central region lighting condition. This overall effect was supported by multiple qualitative comments from our participants such as "The darkness made the experience feel more immersive." This might be related to the task that the participants experienced involved a bit creepy virtual characters, which make the dark environment more appropriate and realistic. Moreover, we received multiple comments from the participants suggesting that the effects of the vibrations were strengthened in the conditions with the central (reduced) lighting that did not reach into the periphery. For instance, one participant commented, "compared to the previous condition, having floor vibrations with lights off felt more immersive than having floor vibrations with lights on." Although this interaction effect is not supported by our statistical tests, it suggests an interesting hypothesis that could be tested in future research.

\subsection{Reduced Peripheral Vision Did Not Change Participants' Experience}

Contrary to our Hypothesis H3, we found no significant effect of the restricted physical field of view on the dependent variables. While we cannot rule out a slight effect of the periphery on our results (see Figure 4), which could be verified with more participants, based on a power analysis we can say that at least it did not have a noticeable (strong) effect on participants' experience. We received informal comments from the participants such as "Didn't notice too much of a difference with the black cloth but I'm sure it helped me focus more on the images of the game." Overall, multiple participants indicated that they were paying attention exclusively to the augmented region of their visual field, which is sometimes called attention funnelling [7], such that the discussed benefits of the restricted periphery (see Section 1) diminished as participants did not (have to) pay attention to the periphery to complete the task. In line with the attention funnelling interpretation, we observed that participants rotated their head more with the HoloLens than what we would expect in a similar real-world situation; previous work suggests that humans may compensate for an attention funnel by adapting their head behavior with an increased amount and frequency of head rotations $[2,14]$. The relatively small size of virtual entities, which the participants could see the entire body of the entity even in a narrow FOV, could also be one of the reasons why they did not pay attention to the periphery. As a guideline for practitioners in $\mathrm{AR}$, we can say that there is no need to restrict the users' peripheral view for tasks that do not require them to pay attention to the wider human visual field.

\section{CONCLUSION AND FUTURE WORK}

In this paper, we presented an analysis of factors and common challenges with OST-HMD setups that can have an impact on users' perceived co-presence when in close proximity of dynamic virtual content and entities. We reported on a human-subject study that we conducted to understand three related factors. First, we evaluated the effects of the unique characteristic of current OST-HMDs that the small augmented visual field and a large unaugmented periphery are in conflict. This can greatly limit a user's perceived impact of a virtual entity when it vanishes out of the virtual field of view while the real world is still visible. We tested a potential compensation based on occluding the entire (real and virtual) periphery. Second, we tested different lighting conditions in the physical environment that were meant to simulate a reduced field of view in line with an occluded periphery. Third, we compared the effects of vibrotactile feedback presented through a physical platform in AR as a means to compensate for the missing visual feedback in the lower periphery. We found that vibrotactile feedback significantly increased spatial and social presence and engagement, and reduced peripheral lighting further increased engagement, while the impact of the peripheral view restriction was limited. 
We believe that it is important to investigate related visual and multimodal technologies that have the potential to increase copresence and reduce perceptual differences between virtual and real stimuli in AR. For future work, we think that it would be important to identify the benefits that larger fields of view provide for future OST-HMDs, as well as further technological means that can create realistic haptic feedback in AR.

\section{ACKNOWLEDGMENTS}

This material includes work supported in part by the National Science Foundation under Award Number 1564065 (Dr. Ephraim P. Glinert, IIS), Award Number 1852002 (Dr. Harriet G. Taylor and Dr. Jonathan M. Sprinkle, CNS), and Collaborative Award Numbers 1800961, 1800947, and 1800922 (Dr. Tonya Smith-Jackson, IIS) to the University of Central Florida, University of Florida, and Stanford University respectively; the Office of Naval Research under Award Number N00014-17-1-2927 (Dr. Peter Squire, Code 34); and the AdventHealth Endowed Chair in Healthcare Simulation (Prof. Welch). Any opinions, findings, and conclusions or recommendations expressed in this material are those of the author(s) and do not necessarily reflect the views of the supporting institutions.

\section{REFERENCES}

[1] P. L. Alfano and G. F. Michel. 1990. Restricting the field of view: perceptual and performance effects. Perceptual and Motor Skills 70, 1 (1990), 35-45.

[2] K. W. Arthur. 2000. Effects of field of view on performance with head-mounted displays. Ph.D. Dissertation. University of North Carolina at Chapel Hill.

[3] J. N. Bailenson, J. Blascovich, A. C. Beall, and J. M. Loomis. 2003. Interpersonal Distances in Virtual Environments. Personality and Social Psychology Bulletin 29 , 7 (2003), 819-833

[4] C. Basdogan, C.-H. Ho, M. A. Srinivasan, and M. Slater. 2000. An experimental study on the role of touch in shared virtual environments. ACM Transactions on Computer-Human Interaction 7, 4 (2000), 443-460.

[5] T. W. Bickmore, R. Fernando, L. Ring, and D. Schulman. 2010. Empathic touch by relational agents. IEEE Transactions on Affective Computing 1, 1 (2010), 60-71.

[6] F. Biocca, C. Harms, and J. Burgoon. 2004. Towards a more robust theory and measure of social presence: Review and suggested criteria. Presence: Teleoperators and Virtual Environments 12, 5 (2004), 456-480.

[7] F. Biocca, C. Owen, A. Tang, and C. Bohil. 2007. Attention issues in spatial information systems: Directing mobile users' visual attention using augmented reality. Fournal of Management Information Systems 23, 4 (2007), 163-184.

[8] J. Blascovich, J. Loomis, A. C. Beall, K. R. Swinth, C. L. Hoyt, and J. N. Bailenson. 2002. TARGET ARTICLE: Immersive Virtual Environment Technology as a Methodological Tool for Social Psychology. Psychological Inquiry 13, 2 (2002), 103-124.

[9] J. Blascovich, J. Loomis, A. C. Beall, K. R. Swinth, C. L. Hoyt, N. Bailenson, and J. N Bailenson. 2002. Immersive Virtual Environment Technology as a Methodological Tool for Social Psychology. Psychological Inquiry 7965 (2002), 103-124.

[10] G. C. Burdea. 1996. Force and touch feedback for virtual reality. John Wiley \& Sons, Inc., New York, USA.

[11] C. Cadena, L. Carlone, H. Carrillo, Y. Latif, D. Scaramuzza, J. Neira, I. Reid, and J. Leonard. 2016. Past, Present, and Future of Simultaneous Localization and Mapping: Toward the Robust-Perception Age. IEEE Transactions on Robotics 32 6 (2016), 1309-1332.

[12] E. H. Calvillo-Gámez, P. Cairns, and A. L. Cox. 2015. Assessing the core elements of the gaming experience. In Game User Experience Evaluation. Springer, 37-62.

[13] P. W. Fink, P. S. Foo, and W. H. Warren. 2007. Obstacle avoidance during walking in real and virtual environments. ACM Transactions on Applied Perception 4, 1 (2007).

[14] J. J. Gallimore, N. G. Brannon, and F. R. Patterson. 1998. The effects of fieldof-view on pilot head movement during low level flight. Human Factors and Ergonomics Society Annual Meeting Proceedings 42, 1 (1998), 6-10.
[15] G. Huisman, J. Kolkmeier, and D. Heylen. 2014. Simulated social touch in a collaborative game. In International Conference on Human Haptic Sensing and Touch Enabled Computer Applications. 248-256.

[16] J. A. Jones, D. M. Krum, and M. T. Bolas. 2016. Vertical field-of-view extension and walking characteristics in head-worn virtual environments. ACM Transactions on Applied Perception (TAP) 14, 2 (2016), 1-9.

[17] J. A. Jones, J. E. Swan, and M. Bolas. 2013. Peripheral Stimulation and its Effect on Perceived Spatial Scale in Virtual Environments. IEEE Transactions on Visualization and Computer Graphics (TVCG) 19, 4 (2013), 701-710.

[18] K. Kim, M. Billinghurst, G. Bruder, H. Duh, and G. F. Welch. 2018. Revisiting Trends in Augmented Reality Research: A Review of the 2nd Decade of ISMAR (2008-2017). IEEE Transactions on Visualization and Computer Graphics (TVCG) 24, 11 (2018), 2947-2962.

[19] T. R Knapp. 1990. Treating ordinal scales as interval scales: an attempt to resolve the controversy. Nursing Research 39, 2 (1990), 121-123.

[20] A. Kotranza, B. Lok, A. Deladisma, C. M. Pugh, and D. S. Lind. 2009. Mixed reality humans: Evaluating behavior, usability, and acceptability. IEEE Transactions on Visualization and Computer Graphics (TVCG) 15, 3 (2009), 369-382.

[21] E. Kruijff, J. E. Swan, and S. Feiner. 2010. Perceptual issues in augmented reality revisited. In Proceedings of the International Symposium on Mixed and Augmented Reality (ISMAR). 3-12.

[22] W. M. Kuzon Jr, M. G. Urbanchek, and S. McCabe. 1996. The seven deadly sins of statistical analysis. Annals of plastic surgery 37, 3 (1996), 265-272.

[23] M. Lee, G. Bruder, T. Höllerer, and G. Welch. 2018. Effects of Unaugmented Periphery and Vibrotactile Feedback on Proxemics with Virtual Humans in AR. IEEE Transactions on Visualization and Computer Graphics (TVCG) 24, 4 (2018), $1525-1534$.

[24] M. Lee, G. Bruder, and G. F. Welch. 2017. Exploring the effect of vibrotactile feedback through the floor on social presence in an immersive virtual environment. IEEE Virtual Reality (VR) (2017), 105-111.

[25] M. Lee, K. Kim, S. Daher, A. Raij, R. Schubert, J. Bailenson, and G. Welch. 2016. The wobbly table: Increased social presence via subtle incidental movement of a real-virtual table. In IEEE Virtual Reality (VR). 11-17.

[26] M. Lee, N. Norouzi, G. Bruder, P. J. Wisniewski, and G. F. Welch. 2018. The physical-virtual table: exploring the effects of a virtual human's physical influence on social interaction. In Proceeding of the ACM Symposium on Virtual Reality Software and Technology (VRST).

[27] M. Lombard, T. B. Ditton, and L. Weinstein. 2009. Measuring Presence: The Temple Presence Inventory. In Proceedings of the Annual International Workshop on Presence. 1-15.

[28] J. M. Loomis and J. M. Knapp. 2003. Visual perception of egocentric distance in real and virtual environments. In Virtual and Adaptive Environments. Number 11. 21-46.

[29] S. Palmer. 1999. Vision Science: Photons to Phenomenology. MIT Press. 1-810 pages.

[30] E. D. Ragan, D. A. Bowman, R. Kopper, C. Stinson, S. Scerbo, and R. P. McMahan. 2015. Effects of Field of View and Visual Complexity on Virtual Reality Training Effectiveness for a Visual Scanning Task. IEEE Transactions on Visualization and Computer Graphics (TVCG) 21, 7 (2015), 794-807.

[31] D. Ren, T. Goldschwendt, Y. Chang, and T. Höllerer. 2016. Evaluating wide-fieldof-view augmented reality with mixed reality simulation. In Proceedings of IEEE Virtual Reality (VR). 93-102.

[32] R. S. Renner, B. M. Velichkovsky, and J. R. Helmert. 2013. The perception of egocentric distances in virtual environments - A review. Comput. Surveys 46, 2 (2013), 1-40.

[33] E.-L. Sallnäs. 2010. Haptic feedback increases perceived social presence. Haptics: Generating and Perceiving Tangible Sensations (Lecture Notes in Computer Science) 6192 (2010), 178-185.

[34] M. Slater. 2009. Place illusion and plausibility can lead to realistic behaviour in immersive virtual environments. Philosophical Transactions of the Royal Society of London B: Biological Sciences 364, 1535 (2009).

[35] A. Toet, S. E. M. Jansen, and N. J. Delleman. 2007. Effects of Field-of-View Restrictions on Speed and Accuracy of Manoeuvring. Perceptual and Motor Skills 105 (2007), 1245-1256.

[36] A. Toet, M. van der Hoeven, M. Kahrimanovic, and N. J. Delleman. 2008. Effects of field of view on human locomotion. In Head-and Helmet-Mounted Displays XIII: Design and Applications, Vol. SPIE-6955. 1-11.

[37] G. Welch, G. Bruder, P. Squire, and R. Schubert. 2019. Anticipating Widespread Augmented Reality: Insights from the 2018 AR Visioning Workshop. Technical Report. University of Central Florida and Office of Naval Research.

[38] S. Zhao. 2003. Toward a taxonomy of copresence. Presence: Teleoperators and Virtual Environments 12, 5 (2003), 445-455. 